\title{
Using the FRAIL scale to compare pre-existing demographic lifestyle and medical risk factors between non-frail, pre-frail and frail older adults accessing primary health care: a cross- sectional study.
}

\author{
Vanessa Aznar-Tortonda ${ }^{1}{ }$, Antonio Palazón-Bru ${ }^{\text {Corresp., }}{ }^{1}$, Vicente F Gil-Guillén ${ }^{1}$ \\ ${ }^{1}$ Department of Clinical Medicine, Miguel Hernández University, San Juan de Alicante, Spain \\ Corresponding Author: Antonio Palazón-Bru \\ Email address: antonio.pb23@gmail.com
}

Background. Few studies in the scientific literature have analyzed frailty status as an ordinal variable (non-frail, pre-frail and frail) rather than as a binary variable (frail versus non-frail). These studies have found that pre-frailty behaves differently from frailty (no ordinality in the variable). However, although the comparison between pre-frail and frail individuals is clinically relevant to understanding how to treat pre-frailty, this comparison was not performed in previous studies.

Materials \& Methods. A cross-sectional observational study was designed with 621 older individuals aged $\geq 60$ years in Spain in 2017-2018, determining factors associated with a higher frailty stage (nonfrail, pre-frail and frail) and undertaking this comparison, in addition to measuring non-frailty. The factors assessed through a multinominal regression model were: age, sex, living alone, recent loss of the partner, income and total comorbidities.

Results. Of the total participants, 285 were non-frail (45.9\%), 210 were pre-frail (33.8\%) and 126 were frail (20.3\%). Compared to non-frail individuals, pre-frail individuals were older, with more comorbidities and a lower income. Compared to non-frail individuals, frail individuals were more likely to be female, older, with more comorbidities and a lower income. Compared to pre-frail individuals, frail individuals were more likely to be female, older and with more comorbidities.

Conclusion. Comparison between the pre-frail and frail groups showed that frail persons were more likely to have a lower income, be female, older and have a higher number of comorbidities. 
1 TITLE PAGE:

2 Title: Using the FRAIL scale to compare pre-existing demographic lifestyle and medical risk

3 factors between non-frail, pre-frail and frail older adults accessing primary health care: a cross-

4 sectional study.

5 Authors: Vanessa Aznar-Tortonda ${ }^{\mathrm{a}}$, Antonio Palazón-Bru ${ }^{\mathrm{a}}$, Vicente F Gil-Guillénª

6 Institution:

7 a. Department of Clinical Medicine, Miguel Hernández University, San Juan de Alicante, 8 Alicante, Spain.

9

10 Corresponding author: Prof. Dr. Antonio Palazón-Bru, Department of Clinical Medicine, Miguel

11 Hernández University, Ctra Valencia-Alicante S/N, 03550 San Juan de Alicante, Alicante, Spain.

12 Telephone number: +34 965919449. E-mail address: antonio.pb23@gmail.com. 


\section{ABSTRACT}

15 Background. Few studies in the scientific literature have analyzed frailty status as an ordinal variable (non-frail, pre-frail and frail) rather than as a binary variable (frail versus non-frail). These studies have found that pre-frailty behaves differently from frailty (no ordinality in the variable). However, although the comparison between pre-frail and frail individuals is clinically relevant to understanding how to treat pre-frailty, this comparison was not performed in previous studies.

Materials \& Methods. A cross-sectional observational study was designed with 621 older individuals aged $\geq 60$ years in Spain in 2017-2018, determining factors associated with a higher frailty stage (non-frail, pre-frail and frail) and undertaking this comparison, in addition to measuring non-frailty. The factors assessed through a multinominal regression model were: age, sex, living alone, recent loss of the partner, income and total comorbidities.

Results. Of the total participants, 285 were non-frail (45.9\%), 210 were pre-frail $(33.8 \%)$ and 126 were frail (20.3\%). Compared to non-frail individuals, pre-frail individuals were older, with more comorbidities and a lower income. Compared to non-frail individuals, frail individuals were more likely to be female, older, with more comorbidities and a lower income. Compared to pre-frail individuals, frail individuals were more likely to be female, older and with more comorbidities.

Conclusion. Comparison between the pre-frail and frail groups showed that frail persons were more likely to have a lower income, be female, older and have a higher number of comorbidities. 


\section{INTRODUCTION}

Frailty is a biological syndrome (Dent et al., 2019), characterized by a state of vulnerability and increased susceptibility to stressful factors due to loss of homeostasis after a stressor event, resulting in the cumulative deterioration of multiple physiological systems (Clegg et al., 2013; Hoogendijk et al., 2019). Frailty is a dynamic state that is not unidirectional, with persons shifting in a continuum, non-frail - pre-frail - frail (Abizanda Soler \& Rodríguez Mañas, 2014).

Frail older people have decreased physiological reserves, which increases their vulnerability and likelihood of adverse health episodes. This is a transition from non-frailty to frailty and then to dependence (Rockwood et al. 1994; Whitson, Purser \& Cohen, 2007; Dent, Kowal \& Hoogendijk, 2016). The last thirty years have seen great, but unsuccessful efforts to find a standard instrument to identify frailty (Hoogendijk et al., 2019). The most used of the many tools to measure frailty is the Phenotype model, described by Fried in 2001. This contemplates five variables: unintentional weight loss, self-reported exhaustion, low energy expenditure, slow gait speed, and weak grip strength. Older adults with three or more factors are considered frail, with one or two factors pre-frail, and with no factors non-frail (Fried et al., 2001; Clegg et al., 2013). Another tool is the FRAIL scale (Fatigue, Resistance, Ambulation, Illnesses, \& Loss of Weight), which considers individuals to be frail if three or more criteria on the scale are met, pre-frail if one or two criteria are met, and non-frail if none are met (Abellan et al., 2008). The FRAIL scale is a brief simple questionnaire comprising five items. It requires no special material or measurements and can therefore be done quickly to identify the state of frailty (Kojima 2018). It has been validated in several populations and is increasingly used in clinical and research settings (Hoogendijk et al., 2019). The Phenotype model of Fried, which has been independently validated, can form the basis for the detection of frailty in daily clinical practice, though it is still 
58 not clear how the variables of the definition can be reliably translated (Clegg et al., 2013). Many

59 other models to measure frailty are also available, such as: Cumulative deficit model, Clinical

60 Frailty Scale, gait speed measurement, the Groningen Frailty Indicator and the Edmonton Frail

61 Scale (Clegg et al., 2013; Hoogendijk et al., 2019).

62 Most medical studies have assessed the presence or absence of frailty, without evaluating pre-

63 frailty (Carneiro et al., 2017). However, recent longitudinal studies have determined that 8.2-

$6418.2 \%$ of older adults in pre-frailty progress to frailty over a mean period of approximately 5

65 years (Doi et al., 2018; Herr et al., 2019; Kojima et al., 2019), with the risk of this transition

66 increasing with exhaustion, physical inactivity, decreased muscle strength and decreased

67 mobility (Doi et al., 2018). Many studies have assessed the factors associated with both frailty

68 and pre-frailty (Abizanda et al., 2011; Hoogendijk et al., 2016; Langholz et al., 2018; Jacobsen et

69 al., 2019), determining whether there is a trend between a greater degree of frailty and each

70 individual factor studied (trend test), that is, considering the stage of frailty to be an ordinal

71 variable. This, however, does not enable us to draw conclusions about possible differences

72 between the stages of frailty. This can be done by comparing the groups independently (e.g., pre-

73 frail vs non-frail) using independent statistical models (binary logistic regression models) or a

74 single model assessing the 3 possible stages jointly (multinomial or ordinal logistic regression

75 models), that is, without leaving out information. Four cross-sectional studies have analyzed

76 frailty status as an ordinal variable (non-frail, pre-frail and frail) rather than as a binary variable

77 (frail vs non-frail) and have studied differences between the groups; one from the United

78 Kingdom (UK), another from Sri Lanka, another from China and the final one from Colombia

79 (Curcio et al., 2014; Hanlon et al., 2018; Ye, Gao \& Fu, 2018; Siriwardhana et al., 2019). The

80 first two studied whether this ordinality made sense through ordinal and multinomial regression 
81 models and saw that the latter have a better fit, i.e., pre-frailty behaves differently than frailty, so

82 it should be considered independently. However, neither study compared pre-frail versus frail

83 individuals, and it is important to ascertain whether different patterns exist between the two

84 stages to consider how to treat a pre-frail person. For this reason, we conducted a study with

85 older people aged 60 or over using a multinomial logistic regression model to determine whether

86 different pre-existing demographic, lifestyle and medical risk factors exist between non-frailty,

87 pre-frailty and frailty (Curcio et al., 2014; Hanlon et al. 2018; Siriwardhana et al., 2019),

88 focusing especially on the comparison between frail and pre-frail individuals. Although the

89 group of pre-frail persons could be considered to have less importance as they have received less

90 attention in previous studies, their prevalence is not small (Curcio et al., 2014; Hanlon et al.,

91 2018; Ye, Gao \& Fu, 2018; Siriwardhana et al., 2019). Bearing in mind that over a period of five

92 years between one in five and one in eight persons pass from pre-frail to frail (Doi et al., 2018;

93 Herr et al., 2019; Kojima et al., 2019), knowing the differences between these groups has great

94 clinical relevance, as it would provide information that can then be used to prevent or delay this

95 situation.

97 MATERIAL AND METHODS

98 Study population, design, participants and ethical considerations

99 The study population included individuals aged 60 or over who visited primary care centers in

100 the Valencian Community (Spain). Health care in Spain is universal and free of charge for all 101 residents. 
102 This cross-sectional observational study analyzed a sample of patients who sought care between

1032017 and 2018 without an appointment at several primary healthcare centers in the Valencian

104 Community: Sagunto and Puerto de Sagunto (Valencia), Monóvar, Las Acacias and Marina

105 Española (Alicante). All patients aged 60 or over visiting these healthcare centers were invited to

106 participate in the study, excluding those who did not expressly wish to participate or who had

107 any physical, psychological or social problem that prevented them from completing the

108 questionnaires that would later be used (see Variables and measurements).

109 The Clinical Research Ethics Committee of Sagunto Hospital and Elda University General

110 Hospital approved the study on March 6, 2017. All participants in the study gave their informed

111 consent in writing.

\section{Variables and measurements}

113 The main study variable was frailty status, which can have three stages in older people: non-frail,

114 pre-frail and frail. This is evaluated with the FRAIL frailty scale, assessing the number of criteria

115 presented by each patient. Each of the criteria (fatigue, resistance, ambulation, illness, and loss of

116 weight) was obtained through a personal interview (Fried et al., 2001; Ferrucci et al., 2004;

117 Rolfson et al., 2006).

118 Secondary variables assessed to determine whether they were risk factors for a higher frailty

119 stage were: age, sex, living alone, recent loss of the partner (less than one year), income level

$120[<$ minimum wage (MW), MW $-1.5 \times \mathrm{MW}$ and $>1.5 \times \mathrm{MW}$; the minimum wage was approximately

121630 euros nationally at the time of data collection] and total number of comorbidities. The sum

122 of the number of comorbidities included: stroke, coronary heart disease, arthrosis or advanced

123 osteoarticular disease, depression, diabetes and chronic obstructive pulmonary disease because 
124 these have shown associations with a higher level of frailty (Hanlon et al., 2018). The

125 justification for selecting these potential factors is that in the three studies assessing frailty as a

126 continuum with three stages (Hanlon et al., 2018; Ye, Gao \& Fu, 2018; Siriwardhana et al.,

127 2019), associations were found with age, sex, marital status and cohabiting. Finally, age was

128 evaluated in a binary manner, setting 80 years as the cut-off point, since it appears in the

129 definition of frailty (Rockwood et al., 1994; Whitson, Purser \& Cohen, 2007; Dent, Kowal \&

130 Hoogendijk, 2016), in addition to being used in previous studies (Siriwardhana et al., 2019), as

131 this is the most frail age group.

\section{Sample size calculation and statistical analysis}

133 The sample size was calculated to estimate the prevalence of pre-frailty and frailty in the older

134 population aged 60 years and older. Expecting to find $39.5 \%$ and $16.9 \%$ of patients with these

135 conditions (Ye, Gao \& Fu, 2018), respectively, setting type I error at 5\% and with an accuracy of

$1364 \%$, at least 574 subjects in total would be needed for pre-frailty and 338 for frailty.

137 Consequently, with 574 patients we could estimate the two proportions with the established

138 error.

139 The qualitative variables were described using absolute and relative frequencies. Income was

140 analyzed as a qualitative variable with the lowest income bracket being set as the reference point.

141 For the single quantitative variable (number of comorbidities), means and standard deviations

142 were calculated if normally distributed, otherwise we used median and interquartile range. The

143 Jonckheere-Terpstra test was used to determine associations between our secondary variables

144 and the main variable (frailty status), the latter being ordinal. We then performed a post-hoc

145 analysis using the Bonferroni correction with the Pearson Chi squared and median tests. Using a 
146 multivariate approach, a multinomial rather than an ordinal logistic regression model was

147 estimated as the literature has shown these to have a different character concerning the behavior

148 of associated factors (Hanlon et al., 2018; Siriwardhana et al., 2019). With this model, the three

149 groups were compared through analysis of the adjusted odds ratios for all the secondary

150 variables. The type I error was 5\% for all analyses, and for each relevant parameter its associated

151 confidence interval (CI) was calculated. The IBM SPSS Statistics 25 statistical package was used

152 for all the calculations.

\section{RESULTS}

Of the 621 older patients included in the study (all those invited agreed to participate), 285 were non-frail (45.9\%, 95\% CI: 42.0-49.8\%), 210 were pre-frail (33.8\%, 95\% CI: 30.1-37.5\%) and

157126 were frail (20.3\%, 95\% CI: $17.1-23.5 \%)$. Regarding the secondary variables (Table 1$)$, in terms of demographic characteristics, it should be noted that there was a greater proportion of women $(58.6 \%)$ and that approximately one in five individuals was over 80 years of age (21.4\%). In addition, one in six individuals lived alone (16.6\%), and 30.1\% had an income below the minimum wage. Finally, the mean number of comorbidities was 1.1 .

Concerning the factors associated with frailty status as a continuum (higher likelihood of being in a more advanced stage of frailty) (Table 1), significant differences $(\mathrm{p}<0.05)$ were observed in all the variables, with the exception of recent loss of the partner $(p=0.391)$. These differences were due to the frailty stage being more advanced in people of older age, women, those living alone at home, and those with a lower level of income. Finally, the number of comorbidities increased as the frailty stage was higher. Analysis within each group of these differences (post- 
168 hoc analysis, Table 2) showed that female sex differed between the frail group and the other two 169 groups, whereas living alone differed between the frail and the non-frail groups. Age and number 170 of comorbidities differed between the three groups, whereas income just differed between the

171 non-frail and the pre-frail groups in the category of higher amounts.

172 When analyzing the results obtained in the multinomial model (Table 3), it is important to note

173 that we had three groups (non-frail, pre-frail and frail) and therefore we had to study two by two

174 comparisons of each of the frailty stages (pre-frail versus non-frail, frail versus non-frail and frail 175 versus pre-frail). Significant differences were found between the pre-frail and the non-frail, with 176 the pre-frail being older, with a greater number of comorbidities and lower incomes. Living 177 alone was non-significant, though the non-frail older patients cohabited more frequently in comparison with the other groups. These same factors were seen when comparing frail and non-

179 frail participants, with the addition of a greater proportion of women in the frail group. Finally, analysis of the differences between the frail and the pre-frail individuals determined that the frail individuals were older, more likely to be women and to have a greater number of comorbidities. After summarizing all the results, we can say that income was the only factor that showed no variation, with an association in the pre-frail and frail groups only when compared with the nonfrail older group. The rest of the factors changed as the frailty stage increased.

\section{DISCUSSION}

Summary

Comparison between pre-frail and frail older individuals shows that the probability of frailty increases as total comorbidities and age increase. Similarly, there was a higher proportion of 
190 women in the most advanced frailty stage. While no differences in income were seen between

191 the pre-frail and frail older individuals, the non-frail group had higher incomes than the other

192 groups, and income is thus a protective factor.

\section{Limitations}

194 Concerning the potential limitations of our study, there may have been selection bias as sampling 195 was consecutive not random, and the sample was taken from the population attending the

196 healthcare center. The results may therefore vary in the general population. Information bias was

197 minimized by using validated questionnaires, analyzing mainly objective variables and ensuring

198 very rigorous data collection, obtaining all data in a primary manner and not through

199 retrospective clinical records. To minimize confounding bias, a multivariate analysis was

200 performed using a model that considers the three frailty stages, which provides better

201 information than assessing frailty alone. Moreover, we analyzed a selective primary care sample,

202 instead of using a random selection, so the participants may not be representative of the

203 population. Nevertheless, we should bear in mind that most patients of this age have chronic

204 disorders and should therefore attend their healthcare center (place where they are treated in

205 Spain) for their physician to control their disorders and prescribe or adapt the medication.

206 Additionally, the number of factors for which associations with frailty was assessed could be

207 limited. Consequently, it would be interesting to address other factors in future studies. We used

208 the stage of frailty as a variable with 3 categories rather than just binary, thereby providing more

209 information. This is important as, when applying mathematical models, no information that is

210 available should be omitted, with quantitative variables to study their functional form and with

211 qualitative variables not to create groups to dichotomize (Moons et al., 2014). Moreover, not

212 having the frailty phenotype data available with performance measures could be a limitation of 
213 the current study, as we used the FRAIL scale. Finally, it would be interesting to obtain

214 demographic characteristics in order to provide a greater chance of finding predictive factors that

215 helped better predict frailty status. For example, variables related to lifetime and recent physical

216 activity, as well as nutritional, alcohol and smoking behaviors would have been useful to include.

217 Additionally, life transitions such as retirement, diagnosis of a major medical condition or

218 moving from one type of residence to another (even from a house to an apartment) might have

219 been useful to assess.

Comparison with the existing literature

221 Concerning the four works that analyzed frailty as an ordinal variable, this measure in three cases

222 was the Phenotype model, with the study in China using, like us, the FRAIL scale (Curcio et al., 223 2014; Hanlon et al., 2018; Ye, Gao \& Fu, 2018; Siriwardhana et al., 2019). Although using the

224 same scale is better when making comparisons, we shall nevertheless attempt to undertake this

225 comparison assuming the limitations.

226 Regarding the prevalence of the three possible frailty stages, $45.9 \%$ of the participants in our

227 study were non-frail. This proportion was higher (59\%) in the study conducted in the UK.

228 However, the UK study sample included individuals aged $\geq 37$ years, which explains these

229 differences (Hanlon et al., 2018). Conversely, the pre-frailty percentage found in this study was

230 similar to our findings and to those of the study from China (Ye, Gao \& Fu, 2018; Siriwardhana

231 et al., 2019). In the case of Sri Lanka and Colombia, though, pre-frailty increased because the

232 studies were conducted in rural areas where there is usually greater pre-frailty and frailty.

233 Finally, the prevalence of frailty in our sample was $20.3 \%$, very similar to the values of the

234 studies from Sri Lanka, Colombia and China (Curcio et al., 2014; Siriwardhana et al., 2019; Ye, 
235 Gao \& Fu, 2018), but considerably higher than the UK study (3\%), probably due to the age cut-

236 off point as a selection criterion (Hanlon et al., 2018).

237 Analysis of the factors associated with the highest frailty stage showed that, unlike in our study,

238 the Chinese study did not compare by groups (pre-frail versus non-frail, frail versus non-frail)

239 and assumed that the factors associated with frailty follow a natural order (ordinal model) (Ye,

240 Gao \& Fu, 2018), whereas the studies from the UK, Colombia and Sri Lanka (Curcio et al., 2014;

241 Hanlon et al., 2018; Siriwardhana et al., 2019), together with our study, use multinomial logistic

242 regression. In addition, the Chinese study evaluated mainly psychosocial factors (Ye, Gao \& Fu,

243 2018), which we have not addressed, making it difficult to compare our results with those of this

244 study.

245 Among the factors studied that were statistically significant in several of the three comparisons

246 (pre-frail versus non-frail, frail versus non-frail, pre-frail versus frail), we found differences in

247 age, sex and income level, as well as in the number of comorbidities. Concerning age and sex,

248 our results are entirely consistent with those of the Chinese study, finding a higher frailty stage

249 with increasing age and in women (Ye, Gao \& Fu, 2018). By contrast, the Sri Lankan study

250 found that only the frail group was older than the non-frail group, with a very strong association

251 from 75 years of age and with very relevant odds ratios (Siriwardhana et al., 2019). However,

252 there were no significant differences in the increase in the number of women in the pre-frail or

253 frail groups compared to the non-frail group (Siriwardhana et al., 2019). In the UK study, the

254 results for age in the pre-frail versus non-frail group are similar and significant, while between

255 the frail and non-frail groups there seems to be a direct association with age, i.e. frailty increases

256 with age. Regarding sex, there were more women in both the pre-frail and frail groups than in the

257 non-frail group. Nevertheless, we should bear in mind that this study did not contemplate only 
258 older individuals (age $\geq 37$ years) (Hanlon et al., 2018). Finally, the results of the Colombia study

259 are fully in agreement with those of our work, as we too found a greater proportion of women

260 and a higher age as the stage of frailty increased (Curcio et al., 2014). When we examine the four

261 studies together, there appears to be a clear association with age in the frail group, but the other

262 associations change according to population, which could be due to sociocultural differences.

263 Another factor studied was income. In the Chinese study, income was not directly assessed,

264 although it seems that the higher the level of education, the lower the frailty stage, and this can

265 therefore be indirectly linked to income (Ye, Gao \& Fu, 2018). In the UK, income was clearly

266 associated with a lower socio-economic level and a higher prevalence of pre-frailty and frailty

267 versus non-frailty (Hanlon et al., 2018). Finally, in the Sri Lankan study, the results concerning

268 income did not reach significance, although an increasing trend was seen between higher frailty

269 stage and lower income (Siriwardhana et al., 2019). In summary, an inverse association between

270 income level and a higher frailty stage seems to be indicated. The final factor assessed was

271 comorbidities, which were only evaluated in the UK and Colombia studies. The former showed

272 that pre-frailty and, even more so, frailty are associated with multimorbidity (Hanlon et al.,

273 2018), as in our study. However, the Columbia study only found differences in this aspect

274 between the frail and the non-frail groups (Curcio et al., 2014). This discrepancy may be because

275 this study grouped the number of comorbidities in a binary form, establishing a cut point of $\geq 3$,

276 which could result in lack of significance.

277 Those studies that just assessed trends (Abizanda et al., 2011; Hoogendijk et al., 2016; Langholz

278 et al., 2018; Jacobsen et al., 2019) mainly used the Fried phenotype. Comparison of their results

279 with the factors also used by us shows total concordance for age, female sex and the number of

280 comorbidities. These studies also assessed various other factors, such as educational level, which 
281 may be related to income, obtaining a similar result to ours. However, other factors studied

282 included clinical and social conditions (attending a healthcare center or hospital, feeling lonely,

283 self-perceived health ...), which are not comparable to the factors evaluated in our study

284 (Abizanda et al., 2011; Hoogendijk et al., 2016; Langholz et al., 2018; Jacobsen et al., 2019).

285 Implications

286 This cross-sectional study found differences between non-frail, pre-frail and frail older adults in 287 sex, age, number of disorders and income. This design opens up other research lines based on 288 our results. The first practical implication concerns screening for frailty. As persons aged 60 289 years or over who have one or more factor found in this study have a higher risk of being frail 290 (woman, older, a higher number of comorbidities and lower income), these persons should be assessed for frailty in primary care. This could enable it to be detected early or even reverse its stage (mainly from frail to pre-frail). Secondly, interventions could be considered based on

293 preventive activities in older persons, focusing particularly on those persons who have some of 294 the factors found here. The aim of these interventions would be to reduce the likelihood of 295 frailty-associated complications (Rockwood et al. 1994; Whitson, Purser \& Cohen, 2007; Dent, 296 Kowal \& Hoogendijk, 2016). Together, these two aspects would result in a better quality of life 297 for older persons attending primary care centers and possibly reduce health care costs.

Among people aged 60 years and over who seek primary care, differences were found in sex, 301 age, income and the number of comorbidities between pre-frail and frail individuals. There was more likelihood of being in the frail group if the individual was a woman, older, had a higher 
303 number of comorbidities and lower income. Consequently, it is of great importance to promote 304 the detection of these conditions so as to prevent frailty early.

305

306 ACKNOWLEDGMENTS

307 The authors thank Maria Repice and Ian Johnstone for their help with the English version of the 308 text. 
309

310 Abellan van Kan G, Rolland YM, Morley JE, Vellas B. 2008. Frailty: toward a clinical 311

312

313

314

315

316

317

318

319

320

321

322

323

324

325

326

327

328

\section{REFERENCES}

definition. J Am Med Dir Assoc 9: 71-72.

Abizanda Soler P, Rodríguez Mañas L. 2014. Evolución histórica del término "Fragilidad".

Realidad actual. In: Ramos Cordero P, ed. Guía de buena práctica clínica en Geriatría.

Fragilidad y nutrición en el anciano. Madrid, Spain: Sociedad Española de Geriatría y

Gerontología.

Abizanda P, Sánchez-Jurado PM, Romero L, Paterna G, Martínez-Sánchez E, Atienzar-Núñez P. 2011. Prevalence of frailty in a Spanish elderly population: the Frailty and Dependence in Albacete study. J Am Geriatr Soc 59: 1356-1359.

Carneiro JA, Cardoso RR, Durães MS, Guedes MCA, Santos FL, Costa FMD, Caldeira AP. 2017. Frailty in the elderly: prevalence and associated factors. Revista Brasileira de Enfermagem 70: 747-752.

Clegg A, Young J, Iliffe S, Rikkert MO, Rockwood K. 2013. Frailty in elderly people. Lancet 381: $752-762$.

Curcio CL, Henao GM, Gomez F. 2014. Frailty among rural elderly adults. BMC Geriatr 14: 2.

Dent E, Kowal P, Hoogendijk EO. 2016. Frailty measurement in research and clinical practice: a review. European Journal of Internal Medicine 31: 3-10.

Dent E, Martin FC, Bergman H, Woo J, Romero-Ortuno R, Walston JD. 2019. Management of frailty: opportunities, challenges, and future directions. Lancet 2019; 394: 1376-1386. 
329 Doi T, Makizako H, Tsutsumimoto K, Nakakubo S, Kim MJ, Kurita S, Hotta R, Shimada H.

330 2018. Transitional status and modifiable risk of frailty in Japanese older adults: A prospective

331 cohort study. Geriatrics \& Gerontology International 18: 1562-1566.

332 Ferrucci L, Guralnik JM, Studenski S, Fried LP, Cutler GB, Walston JD. 2004. Designing

333 randomized, controlled trials aimed at preventing or delaying functional decline and disability in

334 frail, older persons: a consensus report. Journal of the American Geriatrics Society 52: 625-634.

335 Fried LP, Tangen CM, Walston J Newman AB, Hirsch C, Gottdiener J, Seeman T, Tracy R, Kop

336 WJ, Burke G, McBurnie MA; Cardiovascular Health Study Collaborative Research Group. 2001.

337 Frailty in older adults: Evidence for a phenotype. The Journals of Gerontology: Series A 56:

338 M146-M156.

339 Hanlon P, Nicholl BI, Jani BD, Lee D, McQueenie R, Mair FS. 2018. Frailty and pre-frailty in 340 middle-aged and older adults and its association with multimorbidity and mortality: a prospective 341 analysis of 493737 UK Biobank participants. Lancet Public Health 3: e323-e332.

342 Herr M, Cesari M, Landre B, Ankri J, Vellas B, Andrieu S; MAPT/DSA Study Group. 2019.

343 Factors associated with changes of the frailty status after age 70: Findings in the MAPT study. 344 Annals of Epidemiology 34: 65-70.e1.

345 Hoogendijk EO, Afilalo J, Ensrud KE, Kowal P, Onder G, Fried LP. 2019. Frailty: implications 346 for clinical practice and public health. Lancet 394: 1365-1375.

347 Hoogendijk EO, Suanet B, Dent E, Deeg DJ, Aartsen MJ. 2016. Adverse effects of frailty on 348 social functioning in older adults: Results from the Longitudinal Aging Study Amsterdam.

349 Maturitas 83: 45-50. 
350 Jacobsen KK, Jepsen R, Lembeck MA, Nilsson C, Holm E. 2019. Associations between the

351 SHARE frailty phenotype and common frailty characteristics: evidence from a large Danish

352 population study. BMJ Open 9: e032597.

353 Kojima G. 2018. Frailty defined by FRAIL scale as a predictor of mortality: a systematic review

354 and meta-analysis. J Am Med Dir Assoc 19: 480-483.

355 Kojima G, Taniguchi Y, Iliffe S, Jivraj S, Walters K. 2019. Transitions between frailty states

356 among community-dwelling older people: A systematic review and meta-analysis. Ageing

357 Research Reviews 50: 81-88.

358 Langholz PL, Strand BH, Cook S, Hopstock LA. 2018. Frailty phenotype and its association

359 with all-cause mortality in community-dwelling Norwegian women and men aged 70 years and

360 older: The Tromsø Study 2001-2016. Geriatr Gerontol Int 18: 1200-1205.

361 Moons KG, de Groot JA, Bouwmeester W, Vergouwe Y, Mallett S, Altman DG, Reitsma JB,

362 Collins GS. 2014. Critical appraisal and data extraction for systematic reviews of prediction

363 modelling studies: the CHARMS checklist. PLoS Med 11: e1001744.

364 Rockwood K, Fox RA, Stolee P, Robertson D, Beattie L. 1994. Frailty in elderly people: an

365 evolving concept. CMAJ 150: 489-495.

366 Rolfson DB, Majumdar SR, Tsuyuki RT, Tahir A, Rockwood K. 2006. Validity and reliability of

367 the Edmonton Frail Scale. Age \& Ageing 35; 526-529.

368 Siriwardhana DD, Weerasinghe MC, Rait G, Falcaro M, Scholes S, Walters KR. 2019.

369 Prevalence of frailty in rural community-dwelling older adults in Kegalle district of Sri Lanka: a

370 population-based cross-sectional study. BMJ Open 9: e026314. 
371 Whitson HE, Purser JL, Cohen HJ. 2007. Frailty thy name is y Phrailty?. The Journals of

372 Gerontology Series A Biological Sciences and Medical Sciences 62: 728-730.

373 Ye B, Gao J, Fu H. 2018. Associations between lifestyle, physical and social environments and

374 frailty among Chinese older people: a multilevel analysis. BMC Geriatrics 18: 314. 


\section{Table $\mathbf{1}$ (on next page)}

Descriptive and bivariate analysis of the factors associated with frailty in the older individuals.

Abbreviations: IQR, interquartile range; MW, minimum wage; $\mathrm{n}(\%)$, absolute frequency (relative frequency). Comorbidities included: stroke, coronary heart disease, arthrosis or advanced osteoarticular disease, depression, diabetes and chronic obstructive pulmonary disease. ${ }^{*}$, Jonckheere-Terpstra test. 
1 Table 1: Descriptive and bivariate analysis of the factors associated with frailty in the older individuals.

\begin{tabular}{|c|c|c|c|c|c|}
\hline Variable & $\begin{array}{c}\text { Total } \\
\mathrm{n}=621 \\
\mathrm{n}(\%) / \operatorname{median}(\mathrm{IQR})\end{array}$ & $\begin{array}{c}\text { Non-frail } \\
\mathrm{n}=285(45.9 \%) \\
\mathrm{n}(\%) / \text { median(IQR) }\end{array}$ & $\begin{array}{c}\text { Pre-frail } \\
\mathrm{n}=210(33.8 \%) \\
\mathrm{n}(\%) / \text { median(IQR) }\end{array}$ & $\begin{array}{c}\text { Frail } \\
\mathrm{n}=126(20.3 \%) \\
\mathrm{n}(\%) / \text { median(IQR) }\end{array}$ & p-value* \\
\hline Female sex & $364(58.6)$ & $148(40.7)$ & $125(34.3)$ & $91(25.0)$ & $<0.001$ \\
\hline Age $>80$ years & $133(21.4)$ & $38(28.6)$ & $48(36.1)$ & $47(35.3)$ & $<0.001$ \\
\hline Living alone & $103(16.6)$ & $34(33.0)$ & $41(39.8)$ & $28(27.2)$ & 0.004 \\
\hline Recent loss of the partner & $11(1.8)$ & $3(27.3)$ & $6(54.5)$ & $2(18.2)$ & 0.391 \\
\hline \multicolumn{6}{|l|}{ Income: } \\
\hline Low $(<\mathrm{MW})$ & $187(30.1)$ & $71(38.0)$ & $69(36.9)$ & $47(25.1)$ & $<0.001$ \\
\hline Medium (MW-1.5×MW) & $228(36.7)$ & $97(42.5)$ & $87(38.2)$ & $44(19.3)$ & \\
\hline $\operatorname{High}(>1.5 \times \mathrm{MW})$ & $206(33.2)$ & $117(56.8)$ & $54(26.2)$ & $35(17.0)$ & \\
\hline Number of comorbidities & $1(2)$ & $1(1)$ & $1(2)$ & $2(1)$ & $<0.001$ \\
\hline
\end{tabular}

2 Abbreviations: IQR, interquartile range; MW, minimum wage; $\mathrm{n}(\%)$, absolute frequency (relative frequency).

3 Comorbidities included: stroke, coronary heart disease, arthrosis or advanced osteoarticular disease, depression, diabetes and chronic

4 obstructive pulmonary disease. ${ }^{*}$, Jonckheere-Terpstra test. 


\section{Table 2 (on next page)}

Post-hoc with the Bonferroni correction of the bivariate analysis of the significant $(p<0.05)$ factors associated with frailty in the older individuals ( $p$-values).

Abbreviations: MW, minimum wage. Comorbidities included: stroke, coronary heart disease, arthrosis or advanced osteoarticular disease, depression, diabetes and chronic obstructive pulmonary disease. 
1 Table 2: Post-hoc with the Bonferroni correction of the bivariate analysis of the significant $(\mathrm{p}<0.05)$ factors associated with frailty in 2 the older individuals (p-values).

\begin{tabular}{|c|c|c|c|c|c|}
\hline Variable & $\begin{array}{c}\text { Pre-frail vs non- } \\
\text { frail }\end{array}$ & $\begin{array}{l}\text { Frail vs non- } \\
\text { frail }\end{array}$ & $\begin{array}{l}\text { Frail vs pre- } \\
\text { frail }\end{array}$ & $\begin{array}{l}\text { Number of } \\
\text { comparisons }\end{array}$ & $\begin{array}{c}\text { Threshold } \mathrm{p} \text {-value for } \\
\text { significance }\end{array}$ \\
\hline Age $>80$ years & 0.006 & $<0.001$ & 0.004 & 3 & 0.017 \\
\hline Income: & & & & \multirow{4}{*}{9} & \multirow{4}{*}{0.0056} \\
\hline Low $(<\mathrm{MW})$ & 0.052 & 0.010 & 0.407 & & \\
\hline Medium (MW-1.5×MW) & 0.093 & 0.862 & 0.236 & & \\
\hline $\operatorname{High}(>1.5 \times \mathrm{MW})$ & $<0.001$ & 0.010 & 0.678 & & \\
\hline Number of comorbidities & $<0.001$ & $<0.001$ & $<0.001$ & 3 & 0.017 \\
\hline
\end{tabular}

3 Abbreviations: MW, minimum wage.

4 Comorbidities included: stroke, coronary heart disease, arthrosis or advanced osteoarticular disease, depression, diabetes and chronic

5 obstructive pulmonary disease. 


\section{Table 3 (on next page)}

Multinomial logistic regression of frailty status among the older individuals.

Abbreviations: Adj. OR, adjusted odds ratio; $\mathrm{Cl}$, confidence interval; $\mathrm{MW}$, minimum wage (€630). Comorbidities included: stroke, coronary heart disease, arthrosis or advanced osteoarticular disease, depression, diabetes and chronic obstructive pulmonary disease. 
1 Table 3: Multinomial logistic regression of frailty status among the older individuals.

\begin{tabular}{|c|c|c|c|c|c|c|}
\hline Variable & $\begin{array}{l}\text { Pre-frail vs non-frail } \\
\text { Adj. OR }(95 \% \mathrm{CI})\end{array}$ & p-value & Frail vs non-frail & p-value & Frail vs pre-frail & p-value \\
\hline Female sex & $1.05(0.71-1.57)$ & 0.804 & $1.89(1.11-3.20)$ & 0.019 & $1.79(1.06-3.03)$ & 0.029 \\
\hline Age $>80$ years & $1.70(1.04-2.78)$ & 0.036 & $3.16(1.79-5.56)$ & $<0.001$ & $1.86(1.10-3.13)$ & 0.020 \\
\hline Living alone & $1.65(0.96-2.84)$ & 0.069 & $1.73(0.90-3.31)$ & 0.099 & $1.05(0.58-1.91)$ & 0.880 \\
\hline Recent loss of the partner & $1.13(0.26-4.92)$ & 0.870 & $0.44(0.07-3.03)$ & 0.408 & $0.39(0.07-2.11)$ & 0.276 \\
\hline \multicolumn{7}{|l|}{ Income: } \\
\hline Low $(<\mathrm{MW})$ & 1 & & 1 & & 1 & \\
\hline Medium (MW-1.5×MW) & $0.86(0.54-1.37)$ & 0.524 & $0.54(0.30-0.98)$ & 0.042 & $0.63(0.36-1.11)$ & 0.109 \\
\hline $\operatorname{High}(>1.5 \times \mathrm{MW})$ & $0.45(0.27-0.74)$ & 0.002 & $0.42(0.22-0.77)$ & 0.005 & $0.93(0.50-1.72)$ & 0.817 \\
\hline Number of comorbidities & $1.79(1.43-2.25)$ & $<0.001$ & $3.12(2.39-4.08)$ & $<0.001$ & $1.74(1.37-2.21)$ & $<0.001$ \\
\hline
\end{tabular}

2 Abbreviations: Adj. OR, adjusted odds ratio; CI, confidence interval; MW, minimum wage (€630).

3 Comorbidities included: stroke, coronary heart disease, arthrosis or advanced osteoarticular disease, depression, diabetes and chronic 4 obstructive pulmonary disease. 